\title{
Azimuthal correlations in high-energy pp/pA collisions and anisotropic gluon distributions
}

\section{Adrian DUMITRU*广}

Baruch College (City University of New York)

E-mail: adrian.dumitrudbaruch. cuny . edu

\begin{abstract}
I discuss recent ideas relating to anisotropic gluon distributions in the strong field limit of highenergy QCD. They lead to anisotropic particle scattering and, hence, to disconnected contributions to multi-particle azimuthal correlations. A first exploratory analysis of the amplitude of the $\cos (2 \phi)$ anisotropy of the gluon distribution at small $x$ is presented.
\end{abstract}

XXIII International Workshop on Deep-Inelastic Scattering

27 April - May 12015

Dallas, Texas

${ }^{*}$ Speaker.

$\dagger$ I thank all my collaborators for their contributions and the organizers of WG2 "Small-x, Diffraction and Vector Mesons" for the opportunity to present this work. I am grateful for support from the DOE Office of Nuclear Physics through Grant No. DE-FG02-09ER41620 and from The City University of New York through the PSC-CUNY Research Award Program, grant 67119-0045. 


\section{Anisotropic gluon distribution at small $x$}

Consider scattering of a (small) dipole in the fundamental representation off a large nucleus. At high energies the S-matrix in the eikonal approximation is given by [1]

$$
\mathscr{S}(\mathbf{r}, \mathbf{b}) \equiv \frac{1}{N_{c}} \operatorname{tr} V^{\dagger}(\mathbf{x}) V(\mathbf{y})
$$

where $\mathbf{r} \equiv \mathbf{x}-\mathbf{y}$ is the dipole vector and $\mathbf{b} \equiv \frac{1}{2}(\mathbf{x}+\mathbf{y})$ is the impact parameter. $V(\mathbf{x})$ denotes a light-like Wilson line describing the propagation of the projectile through the field of the target

$$
V(\mathbf{x})=\mathbb{P} \exp \left\{i g \int \mathrm{d} x^{-} A^{+a}\left(x^{-}, \mathbf{x}\right) t^{a}\right\}
$$

The stochastic nature [2] of the small- $x$ target field $A^{+a}\left(x^{-}, \mathbf{x}\right)$ gives rise to a non-trivial angular dependence of $\mathscr{S}(\mathbf{r})$ under rotations of $\mathbf{r}[3,4]$.

In the MV model [2] the large- $x$ valence partons act as random, recoilless color charges $\rho^{a}(\mathbf{x})$ described by the effective action

$$
S_{\text {eff }}\left[\rho^{a}\right]=\int \mathrm{d} x^{-} \mathrm{d}^{2} \mathbf{x} \frac{\rho^{a}\left(x^{-}, \mathbf{x}\right) \rho^{a}\left(x^{-}, \mathbf{x}\right)}{2 \mu^{2}}
$$

with $\mu^{2} \sim g^{2} A^{1 / 3}$ proportional to the thickness of a nucleus and to the so called saturation scale $Q_{s}^{2} \sim g^{4} \mu^{2}$. The Weizsäcker-Williams fields generated by $\rho^{a}(\mathbf{x})$ are pure gauges; in covariant gauge,

$$
A^{\mu a}\left(x^{-}, \mathbf{x}\right)=-\delta^{\mu+} \frac{g}{\nabla^{2}} \rho^{a}\left(x^{-}, \mathbf{x}\right) .
$$

Using Eq. (1.4) in Eqs. $(1.1,1.2)$ provides the S-matrix for each target configuration.

The random distribution of color charges $\rho^{a}(\mathbf{x})$ will clearly generate azimuthally anisotropic soft fields $A^{\mu a}\left(x^{-}, \mathbf{x}\right)$. The angular structure fluctuates from one configuration $\rho^{a}(\mathbf{x})$ of valence charges to the next. Averaging over these fluctuations projects onto the isotropic part of the gluon distribution,

$$
\frac{g^{2}}{N_{c}}\left\langle E_{i}^{a}\left(\mathbf{b}_{1}\right) E_{j}^{b}\left(\mathbf{b}_{2}\right)\right\rangle=\frac{1}{N_{c}^{2}-1} \delta^{a b} \delta_{i j} Q_{s}^{2} \Delta\left(\mathbf{b}_{1}-\mathbf{b}_{2}\right) .
$$

$\Delta(\mathbf{b})$ describes the impact parameter dependence of the correlator; its explicit form is not important here. The light-cone electric field of the target in covariant gauge is given by

$$
E^{i}(\mathbf{b})=\int d x^{-} F^{+i}=-\partial^{i} \int d x^{-} A^{+}\left(x^{-}, \mathbf{b}\right) .
$$

However, the MV model sources $\rho^{a}(\mathbf{x})$ are slow variables, and so are their angular fluctuations. Hence, they should be averaged over only after the final observables such as $c_{n}\{2\}$ (see below) have been computed. Hence, observables that probe the angular fluctuations of the stochastic fields would be described by an electric field correlator of the form [5]

$$
\frac{g^{2}}{N_{c}}\left\langle E_{i}^{a}\left(\mathbf{b}_{1}\right) E_{j}^{b}\left(\mathbf{b}_{2}\right)\right\rangle_{\hat{a}}=\frac{1}{N_{c}^{2}-1} \delta^{a b} Q_{s}^{2} \Delta\left(\mathbf{b}_{1}-\mathbf{b}_{2}\right)\left(\delta_{i j}+2 A_{2}\left[\hat{a}_{i} \hat{a}_{j}-\frac{1}{2} \delta_{i j}\right]\right) .
$$

This applies if the amplitude of $\cos 2 \phi$ anisotropies dominates. 

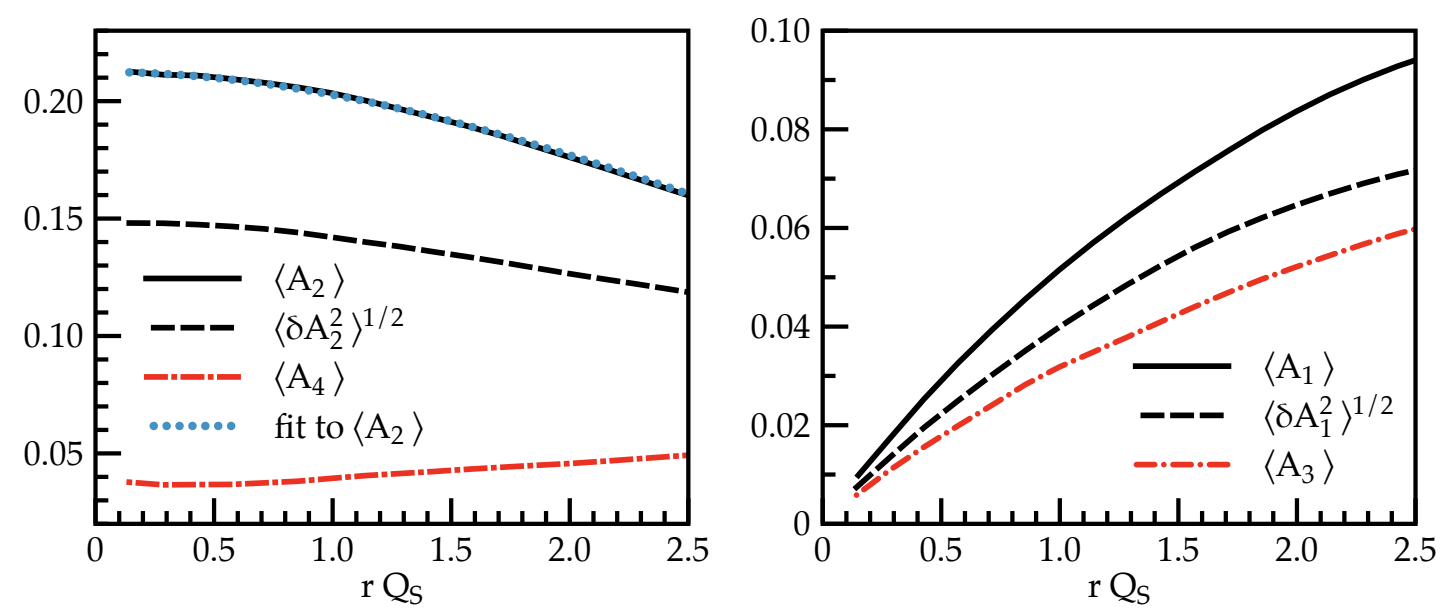

Figure 1: The $\sim \cos n \phi$ amplitudes $\left\langle A_{n}\right\rangle(r)$ as functions of the dipole size $r$ for $n=1, \cdots, 4$. The fit to $\left\langle A_{2}\right\rangle$ corresponds to the function from Eq. (1.14). Figure from ref. [4].

For a general configuration of the sources, the S-matrix for a fundamental charge and $N_{c} \geq 3$ is complex. The real (imaginary) part corresponds to $\mathscr{C}$-even ( $\mathscr{C}$-odd) interactions [6]:

$$
\begin{aligned}
1-D(\mathbf{r}) & \equiv \operatorname{Re} \mathscr{S}(\mathbf{r})=\frac{1}{2 N_{c}} \operatorname{tr}\left[V^{\dagger}(\mathbf{x}) V(\mathbf{y})+V^{\dagger}(\mathbf{y}) V(\mathbf{x})\right], \\
O(\mathbf{r}) & \equiv \operatorname{Im} \mathscr{S}(\mathbf{r})=\frac{-i}{2 N_{c}} \operatorname{tr}\left[V^{\dagger}(\mathbf{x}) V(\mathbf{y})-V^{\dagger}(\mathbf{y}) V(\mathbf{x})\right] .
\end{aligned}
$$

The azimuthal amplitudes can be extracted by expanding the real and imaginary parts of the Smatrix in a Fourier series:

$$
\begin{aligned}
& D(\mathbf{r})=\mathscr{N}(r)\left(1+\sum_{n=1}^{\infty} A_{2 n}^{\prime}(r) \cos \left(2 n \varphi_{r}\right)\right), \\
& O(\mathbf{r})=\mathscr{N}(r) \sum_{n=0}^{\infty} A_{2 n+1}^{\prime}(r) \cos \left[(2 n+1) \varphi_{r}\right] .
\end{aligned}
$$

Here, the function $\mathscr{N}(r)$ denotes the isotropic part of the dipole S-matrix. As already mentioned above each amplitude $A_{n}^{\prime}$ contains a random phase which fluctuates from configuration to configuration. To discard this phase define $A_{n}=\frac{\pi}{2}\left|A_{n}^{\prime}\right|$; the normalization factor arises from

$$
\frac{\pi}{2} \int \frac{d \psi}{2 \pi}|\cos n \psi|=1 .
$$

These anisotropy amplitudes can finally be averaged over many configurations to obtain $\left\langle A_{1}\right\rangle, \cdots$, $\left\langle A_{4}\right\rangle$, c.f. Fig. 1.

At $r \lesssim 1 / Q_{s}$ the amplitude $\left\langle A_{2}\right\rangle$ is around $20 \%$. Such values are in the range of the asymmetries relevant for phenomenology of pA collisions at collider energies (see below). On the other hand, one should keep in mind that here no attempt has been made to bias the configurations towards "high multiplicities" as done in the experiments. Fig. 1 also shows that the fluctuations $\sqrt{ }\left\langle\left(\delta A_{2}\right)^{2}\right\rangle$ are similar in magnitude to the mean value $\left\langle A_{2}\right\rangle$. Hence, there are rather large fluctuations of $A_{2}$ for different configurations. 

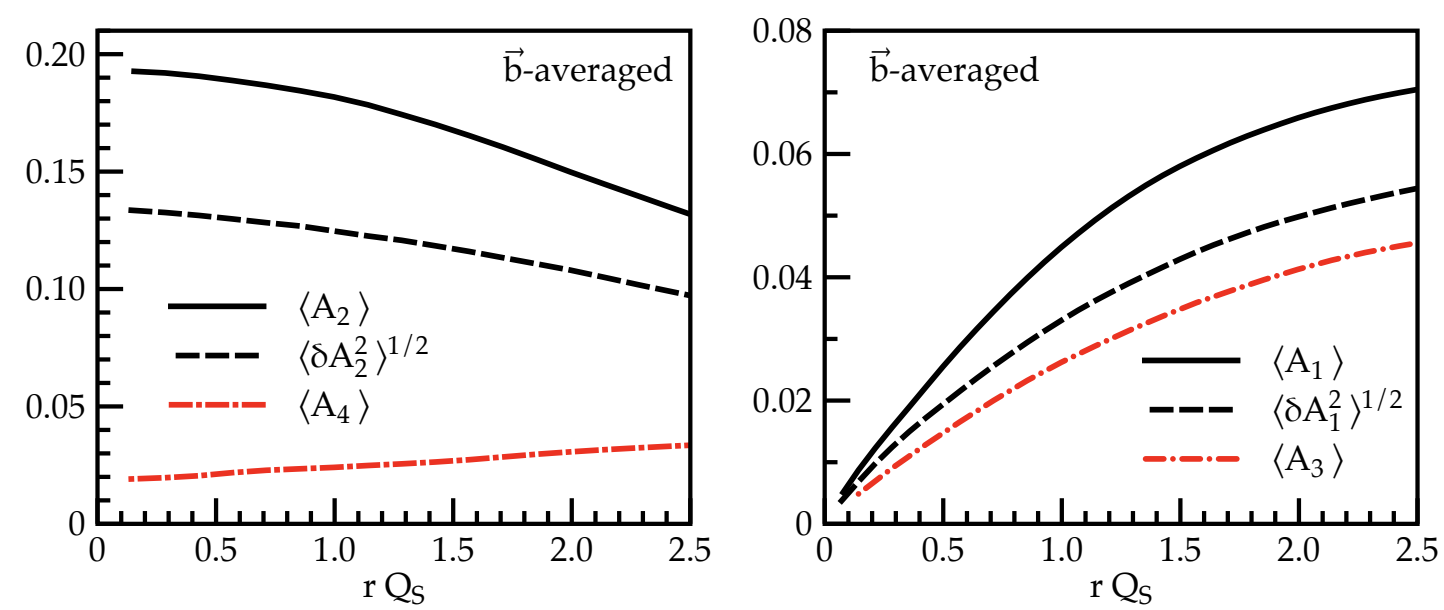

Figure 2: Same as Fig. 1 for "b-smeared" target E-fields [4].

Figure 2 shows the same amplitudes but for $\mathbf{E}$-fields which have been "smeared" over an area $\pi r^{2}$ set by the size of the dipole. Comparing Figs. 1 and 2 one observes that "smearing" has a negligible effect for $r \lesssim 1 / Q_{s}$ while the anisotropy amplitudes at large $r$ are suppressed. This shows the correlation over finite transverse distance scales of the angular structure of the $\mathbf{E}(\mathbf{x})$ configurations.

The MV-model amplitude $\left\langle A_{2}\right\rangle(r)$ matches the distribution of linearly polarized gluons (for an unpolarized target) $h_{1}^{\perp g}\left(x, \mathbf{k}^{2}\right)$ introduced in TMD factorization [7]

$$
\delta^{i j} f_{1}^{g}\left(x, \mathbf{k}^{2}\right)+\left(\hat{k}^{i} \hat{k}^{j}-\frac{1}{2} \delta^{i j}\right) h_{1}^{\perp g}\left(x, \mathbf{k}^{2}\right) .
$$

Within the framework of the MV model, the result for $h_{1}^{\perp g}(x, r)$ derived analytically in Ref. [7],

$$
h_{1}^{\perp g}\left(x, \mathbf{r}^{2}\right) \propto \frac{1}{r^{2} Q_{s}^{2}}\left[1-\exp \left(-\frac{r^{2} Q_{s}^{2}}{4}\right)\right],
$$

is in good agreement with the numerical results from ref [4] at small $r \lesssim 2 Q_{s}^{-1}$.

Fluctuations of the small- $x$ fields in impact parameter space generate a non-zero $\mathscr{C}$-odd component $O(\mathbf{r})$ [8] despite the fact that for the $\mathscr{C}$-even action (1.3) the expectation value $\langle O(\mathbf{r})\rangle=0$. This results in non-zero odd-index amplitudes $A_{1}$ and $A_{3}$, see Fig. 1 . The figure also shows that the expectation values of the odd amplitudes vanish as $r \rightarrow 0$; this is expected because

$$
i O(\mathbf{r}) \sim i \alpha_{s} \mathbf{r} \cdot \nabla_{\mathbf{b}}(1-D(\mathbf{r}, \mathbf{b})) \simeq i \alpha_{s} r^{3} Q_{s}^{2} Q_{c} \cos \varphi_{r}\left[1-\frac{r^{2}}{4}\left(\frac{Q_{c}^{2} \cos ^{2} \varphi_{r}}{3}+Q_{s}^{2}\right)\right]
$$

starts at order $\sim r^{3}$ (three gluon exchange) while in eq. (1.11) we factor out the two-gluon exchange amplitude $\mathscr{N}(r) \sim r^{2}$. The expression on the r.h.s. of (1.15) arises from a gradient expansion in powers of $r$, assuming a generic spectrum of fluctuations of $Q_{s}(\mathbf{b})$ cut off at $Q_{c}$ [9].

The MV model is commonly assumed to describe the gluon distribution of a large nucleus at about $x_{0} \sim 0.01$ in a regime where dipole scattering is coherent but where quantum evolution effects can be neglected. Small- $x$ evolution resums contributions of order $\left(\alpha_{s} \log x_{0} / x\right)^{n}$ to observables. 
Kovner and Lublinsky have solved [3] the Balitsky-Kovchegov evolution equation with explicit azimuthal dependence of the dipole S-matrix but without impact parameter dependence, i.e. $\mathscr{S}(\mathbf{r})$ is allowed to depend on the direction of $\mathbf{r}$ but not on $\mathbf{b}$. They found that evolution to $x<x_{0}$ washes out the anisotropies from the MV model initial condition rather quickly. Indeed, opening up phase space for radiation increases the number of gluons per unit transverse area while bindependent emissions can not induce additional fluctuations and so the gluon distribution should in fact become rather isotropic, quickly. On the other hand, for impact parameter dependent evolution the additional gluons are emitted at random locations in $\mathbf{b}$ space and do not lead to a very rapid "isotropization" of the target electric field [4].

\section{Multi-particle azimuthal correlations}

In the previous section we have argued that the one-particle S-matrix for any particular target configuration is (azimuthally) anisotropic. The vector $\hat{a}$ that spontaneously breaks $2 \mathrm{D}$ rotational symmetry fluctuates randomly from configuration to configuration and can not be measured experimentally. Instead one considers multi-particle azimuthal correlation functions [10] such as

$$
\begin{aligned}
& c_{n}\{2\}=\left\langle e^{i n\left(\phi_{1}-\phi_{2}\right)}\right\rangle, \\
& c_{n}\{4\}=\left\langle e^{i n\left(\phi_{1}+\phi_{2}-\phi_{3}-\phi_{4}\right)}\right\rangle-2\left\langle e^{i n\left(\phi_{1}-\phi_{3}\right)}\right\rangle\left\langle e^{i n\left(\phi_{2}-\phi_{4}\right)}\right\rangle .
\end{aligned}
$$

By definition these cumulants are invariant under global rotations of all particle transverse momenta by the same angle. The anisotropy of the one-particle distribution implies non-zero disconnected contributions to these correlation functions, for example

$$
c_{2}\{2\}=\left\langle e^{2 i \phi_{1}}\right\rangle\left\langle e^{-2 i \phi_{2}}\right\rangle+\left\langle e^{2 i\left(\phi_{1}-\phi_{2}\right)}\right\rangle_{\text {conn. }} .
$$

The product of single-particle anisotropies on the r.h.s. of this equation is independent of the global rotational symmetry breaking vector $\hat{a}$ and so does not vanish upon integration over its directions. In the limit of a strongly anisotropic target gluon distribution, $\left\langle A_{2}\right\rangle=\mathscr{O}(1)$, the $m$-particle cumulants $c_{2}\{m\}$ are in fact dominated by the fully disconnected contribution so that $\left(c_{2}\{m\}\right)^{1 / m} \simeq\left\langle A_{2}\right\rangle$ is the same for all $m$ [5]. The $p_{T}$ dependence of the cumulants in this limit has been worked out analytically within a simple model in ref. [9]; subsequently, Lappi has presented exact numerical results for $c_{2}\{2\}\left(p_{T}\right)$ and $c_{3}\{2\}\left(p_{T}\right)$ which include both disconnected and connected contributions [11]. Qualitatively, his results exhibit a $p_{T}$ dependence which resembles the data from $\mathrm{p}+\mathrm{Pb}$ collisions at the LHC [12] quite well.

In the limit of small $r$ (high $p_{T}$ for all particles) one finds that [5]

$$
\begin{aligned}
& c_{2}\{2\} \propto A_{2}^{2}+\frac{1}{4\left(N_{c}^{2}-1\right)}, \\
& c_{2}\{4\} \propto-A_{2}^{4}+\frac{1}{4\left(N_{c}^{2}-1\right)^{3}} .
\end{aligned}
$$

Thus, both disconnected and connected contributions to $c_{2}\{2\}$ are positive. On the other hand, they enter $c_{2}\{4\}$ with opposite signs; the negative sign of the single particle elliptic deformation (raised to the fourth power) is easily understood from eq. (2.2) when all correlators are assumed 
to factorize. A negative $c_{2}\{4\}$ has been observed by ALICE and CMS in high multiplicity $\mathrm{p}+\mathrm{Pb}$ collisions at the LHC ${ }^{1}$. For the QCD dipole interaction with a static (on the light cone) target electric field,

$$
\frac{1}{N_{c}} \operatorname{tr} V^{\dagger}(\mathbf{x}) V(\mathbf{y})=1+\frac{(i g)^{2}}{2 N_{c}} \operatorname{tr}(\mathbf{r} \cdot \mathbf{E}(\mathbf{b}))^{2}+\cdots,
$$

$c_{2}\{4\}<0$ can arise only in the presence of anisotropic single particle scattering, i.e. for an anisotropic target gluon distribution.

Finally, we return to odd-index cumulants such as $c_{3}\{2\}$. We have seen above that the Fourier harmonics of the target fields obtained from the S-matrix of a single fundamental dipole do contain non-zero odd-index amplitudes $A_{1}$ and $A_{3}$. Their $p_{T}$ dependence has been discussed in refs. [9, 11]. However, experimentally one does not measure Fourier harmonics of the one-particle azimuthal distribution but two (or more) particle cumulants as defined in $(2.1,2.2)$. Lappi has in fact computed the two-particle cumulant $c_{3}\{2\}$ [11] for scattering of a definite two-particle system (say, two quarks). If the two particle S-matrix is summed over $q q, q \bar{q}, \bar{q} q$ and $\bar{q} \bar{q}$ channels though the resulting two-particle S-matrix is

$$
S_{2} \propto\left(\operatorname{tr} V^{\dagger}\left(\mathbf{x}_{1}\right) V\left(\mathbf{y}_{1}\right)+\operatorname{tr} V\left(\mathbf{x}_{1}\right) V^{\dagger}\left(\mathbf{y}_{1}\right)\right)\left(\operatorname{tr} V^{\dagger}\left(\mathbf{x}_{2}\right) V\left(\mathbf{y}_{2}\right)+\operatorname{tr} V\left(\mathbf{x}_{2}\right) V^{\dagger}\left(\mathbf{y}_{2}\right)\right) .
$$

This expression is $\mathscr{C}$-even (real) and so if one assumes quark-anti-quark symmetry of the projectile wave function at small $x$ it has even cumulants, only. Furthermore, the S-matrix for an adjoint projectile charge is real even for scattering of a single particle and so can not generate non-zero $c_{1}\{2\}$ and $c_{3}\{2\}$ as observed experimentally [12].

Therefore, obtaining non-zero $c_{1}\{2\}$ and $c_{3}\{2\}$ may require to account for additional soft rescattering beyond the hard scattering from the target shockwave. Classical Yang-Mills evolution in time of the liberated gluons indeed leads to non-zero $c_{3}\{2\}$ already at a time $\tau \simeq 1 / Q_{s}$ [13]. If such rescattering is soft then the $p_{T}$-distribution of $c_{1}$ and $c_{3}$ from the initial hard scattering should be mostly preserved. A detailed understanding of the numerical results [13] for $c_{3}\{2\}$ is currently lacking.

\section{References}

[1] A. H. Mueller, Nucl. Phys. B 415, 373 (1994); Nucl. Phys. B 437, 107 (1995); A. H. Mueller and B. Patel, Nucl. Phys. B 425, 471 (1994); I. Balitsky, Nucl. Phys. B 463, 99 (1996).

[2] L. D. McLerran and R. Venugopalan, Phys. Rev. D 49, 2233 (1994); Phys. Rev. D 49, 3352 (1994); Y. V. Kovchegov, Phys. Rev. D 54, 5463 (1996).

[3] A. Kovner and M. Lublinsky, Phys. Rev. D 83, 034017 (2011); Phys. Rev. D 84, 094011 (2011); Int. J. Mod. Phys. E 22, 1330001 (2013).

[4] A. Dumitru and V. Skokov, Phys. Rev. D 91, no. 7, 074006 (2015).

[5] A. Dumitru, L. McLerran and V. Skokov, Phys. Lett. B 743, 134 (2015).

[6] Y. Hatta, E. Iancu, K. Itakura and L. McLerran, Nucl. Phys. A 760, 172 (2005).

[7] A. Metz and J. Zhou, Phys. Rev. D 84, 051503 (2011); F. Dominguez, J. W. Qiu, B. W. Xiao and F. Yuan, Phys. Rev. D 85, 045003 (2012).

\footnotetext{
${ }^{1}$ The data for $c_{2}\{4\}$ [12] is integrated over all $p_{T}$ and so can not be explained quantitatively by assuming high $p_{T}$.
} 
[8] Y. V. Kovchegov and M. D. Sievert, Phys. Rev. D 86, 034028 (2012) [Erratum-ibid. D 86, 079906 (2012)].

[9] A. Dumitru and A. V. Giannini, Nucl. Phys. A 933, 212 (2015).

[10] N. Borghini, P. M. Dinh and J. Y. Ollitrault, Phys. Rev. C 63, 054906 (2001).

[11] T. Lappi, Phys. Lett. B 744, 315 (2015).

[12] B. Abelev et al. [ALICE Collaboration], Phys. Lett. B 719, 29 (2013); Phys. Rev. C 90, no. 5, 054901 (2014); G. Aad et al. [ATLAS Collaboration], Phys. Rev. Lett. 110, 182302 (2013); Phys. Lett. B 725, 60 (2013); S. Chatrchyan et al. [CMS Collaboration], Phys. Lett. B 718, 795 (2013); Phys. Lett. B 724, 213 (2013).

[13] B. Schenke, S. Schlichting and R. Venugopalan, Phys. Lett. B 747, 76 (2015). 